\title{
Optical Constants and The Mechanism of the Propagation of the Surface Waves for Silver Films-in View of the Free Electron Model-Using ATR
}

\author{
A.M. Gadou and M.A. El-Sherbiny* \\ Faculty of Science, Mansoura Univ., Mansoura, Egypt \\ *Faculty of Science, Al-Azhar Univ., Cairo, Egypt
}

Using the Drude-free electron theory the polarization parameter $P$ due to interband transitions as well as the optical mass $m_{o}$ and the relaxation time $\tau$ of the electrons could be obtained using the estimated values of the real-and imaginary - part of the dielectric constant $\varepsilon_{r}, \varepsilon_{i}$ estimated by obtained attenuated total reflection-measurements for the boundary Ag/air. It has been clear demonstrated that our estimated values $P, m_{o}, \tau$ are in consistent with published data. This circumstance has the consequence to obtain a general identities to estimate $\varepsilon_{\mathrm{r}}^{\mathrm{f}}, \varepsilon_{\mathrm{i}}^{\mathrm{f}}$ for silver films in a comparatively wide spectral range. Furthermore the limit energy for the surface plasma oscillations $\hbar \omega_{s}=\hbar \omega_{p} / \sqrt{1+\mathrm{P}}=3.66 \mathrm{eV}$ corresponds to infinite values of the $\boldsymbol{K}$-vector of the surface plasma waves. In this case both the phase - and the group - velocity $V_{p}, V_{g}$ of the surface waves must be equal to zero. In addition the energy limit of interband transitions could be estimated as $3.974 \mathrm{eV}$. A derivation of two formulae for the phase- and group- velocity of the surface waves could be obtained from the dispersion relation $(\omega, \mathrm{k})$. In addition the energetic parameter $\beta$ and the wave-number parameter $\chi$ could be estimated with good accuracy. 


\section{Introduction}

It is well known that the frequency of the surface plasma waves propagated at the interface metal-vacuum equals $\omega_{\mathrm{p}} / \sqrt{2}\left(\omega_{\mathrm{p}}\right.$ is the volume plasma frequency). The topics of the surface waves have been demonstrated in some details by $[1,2,3,4]$. In the case of silver, considering the polarization parameter $\mathrm{P}$ due to interband transitions [5], it follows that the limit frequency of the surface waves:

$$
\omega_{\mathrm{s}}=\omega_{\mathrm{p}} / \sqrt{1+\mathrm{P}}
$$

The predicted value,[5], of $\mathrm{P}$ for silver (5.62), is in good agreement with our calculated value of $\mathrm{P} \sim 5.8$. In this case the spectral range for the excitation of the surface plasma waves must be restricted by $\omega<\omega_{\mathrm{s}}$. Surface plasmons are from the optical point of view surface waves, i.e. waves having an amplitude which decreases exponentially on both sides of the surface and which are propagating along the surface as evanescent waves $[5,6]$. Obtained values (by ATR) of the real- and imaginary - parts of the dielectric constant $\varepsilon_{\mathrm{r}}, \varepsilon_{\mathrm{i}}$ were used to estimate $\mathrm{P}, \tau, \mathrm{m}_{\mathrm{o}}$. Agreement with published data was reasonable $[5,7,8]$.

From the dispersion relation $(\omega, \mathrm{k})$ and by using a new identity the values of the energetic parameter $\beta$ and the wave-number parameter $\chi$ could be obtained with good accuracy.

\section{Experimental work}

Silver thin films of thickness $\mathrm{d} \sim 50 \mathrm{~nm}$ were prepared by thermal evaporation onto flint glass substrates under vacuum of less than $10^{-5}$ Torr Kretschmann configuration was used, the experimental details is illustrated elsewhere $[9,10]$. ATR-resonance signals in the investigated spectral range using angular scan method are illustrated in Fig. (1). The resonance angle $\alpha$ was estimated and the real part of the dielectric constant was evaluated using the formula [9].

$$
\left|\varepsilon_{\mathrm{r}}\right|=\frac{\varepsilon_{\mathrm{o}} \varepsilon_{2} \sin ^{2} \alpha}{\varepsilon_{2} \sin ^{2} \alpha-\varepsilon_{\mathrm{o}}}
$$

In the present case $\varepsilon_{0}=1$ where the adjacent medium to the investigated sample is the air and $\varepsilon_{2}$ is obtained from the dispersion curve of the used flint glass prism [9]. 


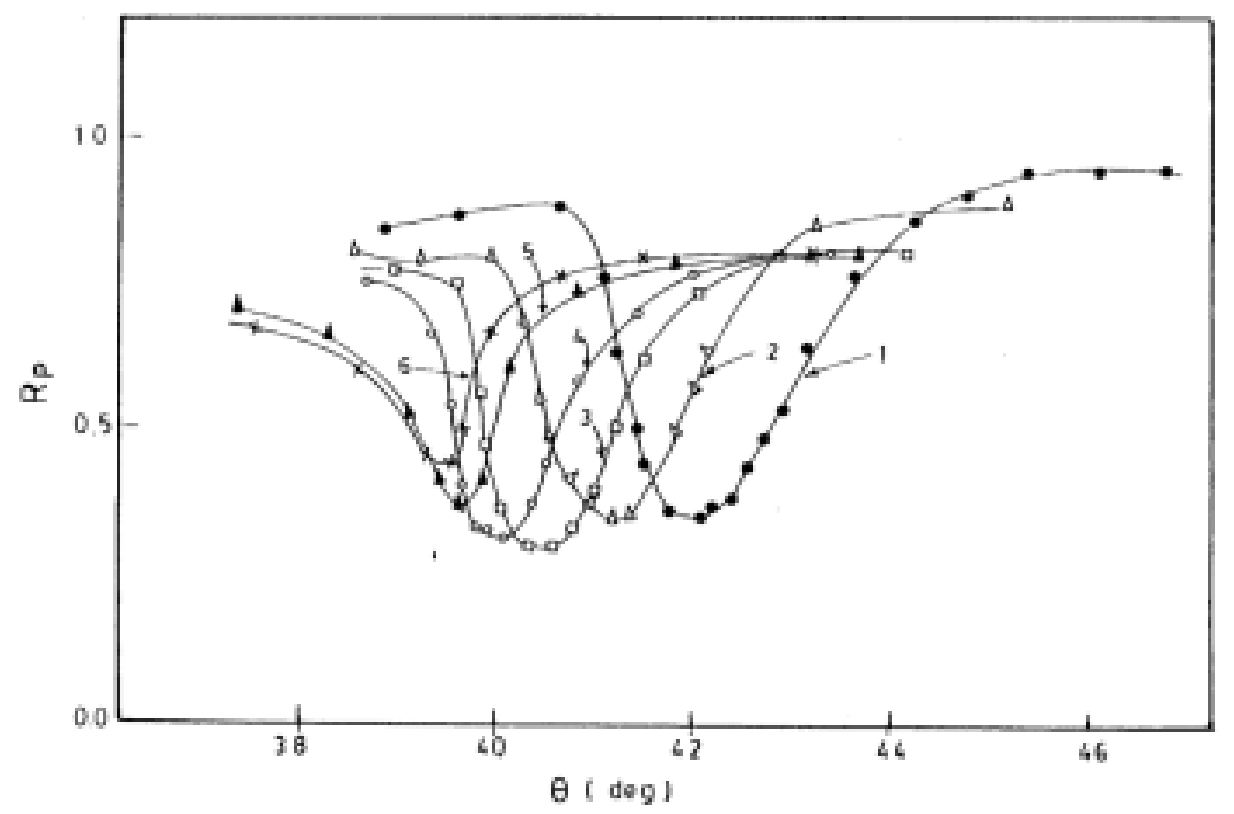

Fig. (1) ATR-resonance signals for the boundary Ag/air d 50 nm 1,2,3,4,5,6 mean signals at $430,460,490,520,550,580 \mathrm{~nm}$ respectively.

It is known that surface plasma waves can be coupled with photons coming from a medium of a relatively high refractive index using angular - or wavelength - Scan $[1,3,6,8]$. For optimum coupling the internal - and radiative damping $\gamma_{\mathrm{i}}, \gamma_{\mathrm{R}}$ are then both equal, wherein the conditions $\varepsilon_{\mathrm{r}}<-1, \varepsilon_{\mathrm{i}} \ll<\left|\varepsilon_{\mathrm{r}}\right|$ must be satisfied $[8,9]$.

$$
\varepsilon_{\mathrm{i}}=\frac{4 \varepsilon_{\mathrm{r}}^{2} \operatorname{Im~r}_{21}}{\left(\left|\varepsilon_{\mathrm{r}}\right|+1\right) \exp \left[\left(\frac{\mathrm{d}}{\lambda}\right) \frac{4 \pi\left|\varepsilon_{\mathrm{r}}\right|}{\sqrt{\left|\varepsilon_{\mathrm{r}}\right|-1}}\right]}
$$

where $r_{21}$ is Fresnel's coefficient.

$$
\operatorname{Im} r_{21}=\frac{2 \varepsilon_{2} \mathrm{a}}{\varepsilon_{2}^{2}+\mathrm{a}^{2}}, \mathrm{a}^{2}=\left|\varepsilon_{\mathrm{r}}\right|\left(\varepsilon_{2}-1\right)-\varepsilon_{2}
$$

\section{Theory}

\section{1- Optical constants and the dispersion of the surface waves:}

The dielectric constant of a metal is determined according to the Drude-free electron theory [5,7] using the values of the optical mass $m_{0}$, the 
relaxation time $\tau$ of the electron and the polarization parameter $\mathrm{P}$ due to interband transitions [5,7] - extracted from our ATR-measurements, as will be immediately shown. It follows that the real- and the imaginary - part of the dielectric constant $\varepsilon_{\mathrm{r}}^{\mathrm{f}}, \varepsilon_{\mathrm{i}}^{\mathrm{f}}$ are given as:

$$
\begin{aligned}
\left|\varepsilon_{\mathrm{r}}^{\mathrm{f}}\right| & \approx \frac{\omega_{\mathrm{p}}^{2} \lambda^{2}}{(2 \pi \mathrm{c})^{2}}-\mathrm{P} \\
\left(\varepsilon_{\mathrm{i}}^{\mathrm{f}} / \lambda\right) & =\frac{\omega_{\mathrm{p}}^{2}}{\tau(2 \pi \mathrm{c})^{3}} \lambda^{2}, \\
\omega_{\mathrm{p}}^{2} & =\frac{4 \pi \mathrm{Ne}^{2}}{\mathrm{~m}_{\mathrm{o}}}
\end{aligned}
$$

where $\mathrm{N}$ is the density of conduction electrons, for silver it is equal to $5.86 \times 10^{22} / \mathrm{cm}^{3}$, and $\lambda$ is the wavelength of the incident photons.

Experimentally measured ATR-resonance signals for the boundary $\mathrm{Ag} /$ air, Fig. (1), were used to estimate $\varepsilon_{\mathrm{r}}, \varepsilon_{\mathrm{i}}$, using eqs.(1\&2). The estimated values are accompanied with errors $\Delta \varepsilon_{\mathrm{r}}, \Delta \varepsilon_{\mathrm{i}}$ which are evaluated using the partial derivative [12]:-

$$
\begin{array}{ll}
\Delta \varepsilon_{\mathrm{r}}=\frac{\partial \varepsilon_{\mathrm{r}}}{\partial \alpha} \cdot \Delta \alpha & \Delta \alpha<<\alpha \\
\Delta \varepsilon_{\mathrm{i}}=\frac{\partial \varepsilon_{\mathrm{i}}}{\partial \mathrm{d}} \cdot \Delta \mathrm{d} & \Delta \mathrm{d}<<\mathrm{d}
\end{array}
$$

It follows that:

$$
\begin{aligned}
& \Delta \varepsilon_{\mathrm{r}}=-\frac{2 \varepsilon_{\mathrm{r}}^{2} \cdot \Delta \alpha}{\varepsilon_{2} \tan \alpha \cdot \sin ^{2} \alpha} \\
& \Delta \varepsilon_{\mathrm{i}}=-\frac{4 \pi\left|\varepsilon_{\mathrm{r}}\right| \varepsilon_{\mathrm{i}}}{\lambda \sqrt{\left|\varepsilon_{\mathrm{r}}\right|-1} \cdot \Delta \mathrm{d}} \\
& \left|\varepsilon_{\mathrm{r}}\right|=\left|\varepsilon_{\mathrm{r}}^{\prime}\right| \pm \Delta \varepsilon_{\mathrm{r}}, \quad \varepsilon_{\mathrm{i}}=\varepsilon_{\mathrm{i}}^{\prime} \pm \Delta \varepsilon_{\mathrm{i}}
\end{aligned}
$$


$\Delta \alpha$ : The statistical error due to the broad signals, Fig. (1). The values of $\Delta \varepsilon_{\mathrm{r}}, \Delta \varepsilon_{\mathrm{i}}$ have been taken into account for errors of the measured layer thickness of the order of $\Delta \mathrm{d} \sim 0.1 \mathrm{~d}=5 \mathrm{~nm}$. Figs. $(2,3)$ show the relation between $\varepsilon_{\mathrm{r}}$ - as well as $\varepsilon_{\mathrm{i}} / \lambda$ - against $\lambda^{2}$. From Fig. (2) and using eq.3, the values of $\mathrm{P}, \omega_{\mathrm{p}}^{2}$ and $\mathrm{m}_{\mathrm{o}}$ could be obtained. Furthermore the value of $\tau$ could be obtained from Fig. (3) using eq. 4. The extracted values listed in Table (1) are in consistent with those obtained by $[5,7]$.

We now use eqs.3,4 in order to estimate $\varepsilon_{\mathrm{r}}^{\mathrm{f}}, \varepsilon_{\mathrm{i}}^{\mathrm{f}}$, in a wide spectral range in comparing to those extracted from our ATR measurements. From our extracted values $p, \omega_{p}^{2}, m_{o}$ and $\tau$ the values of $\varepsilon_{\mathrm{r}}^{\mathrm{f}}, \varepsilon_{\mathrm{i}}^{\mathrm{f}}$ could be estimated as shown in Figs $(4,5)$ which are in accordance with those obtained by using ATR measurements [8] and by extracted from reflection and transmission spectra $[5,7]$. The inequality of the effective mass $m_{0}$ with the free electron mass $m$ is due to the deviation of the
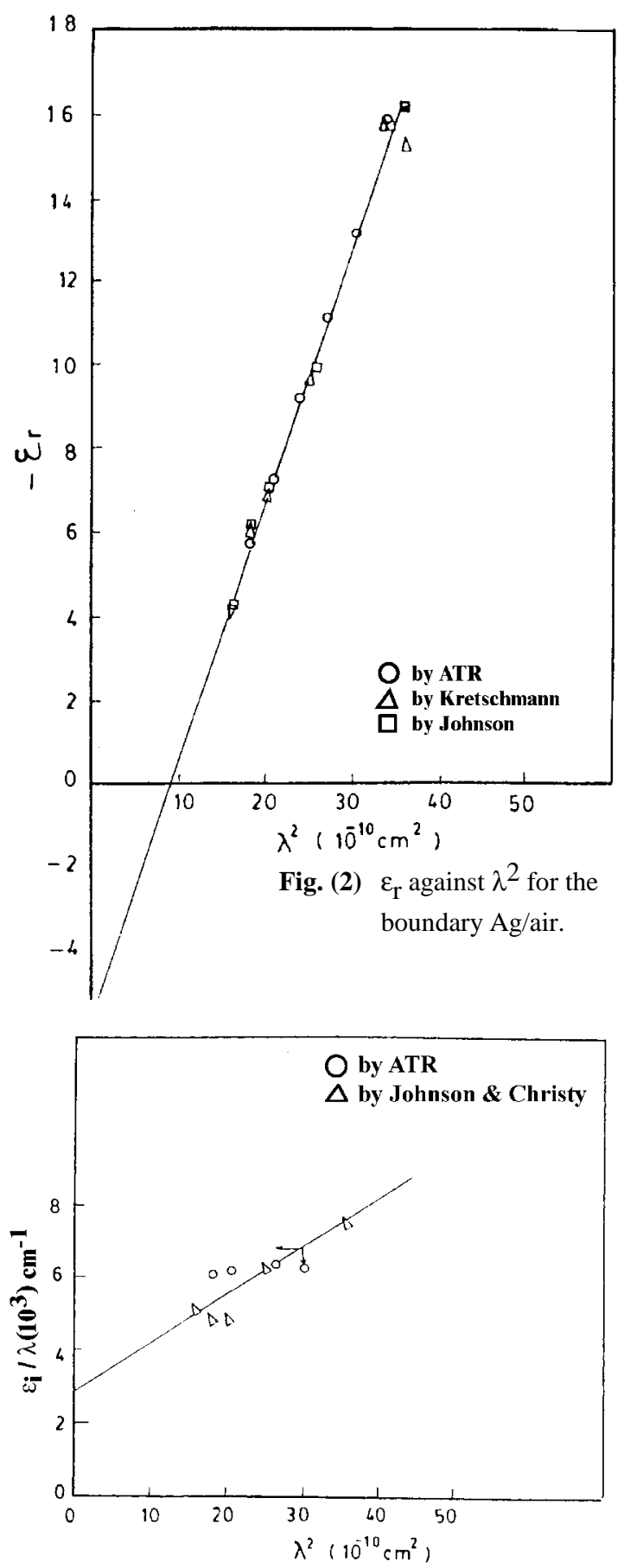

Fig. (3) $\varepsilon_{\mathrm{i}} / \lambda$ against $\lambda^{2}$ for the boundary Ag/air. 
effective number of valence electrons $\mathrm{n}_{\text {eff }}$ per atom from unity at low photon energies [13].

We now utilize our extracted values $\varepsilon_{\mathrm{r}}^{(\mathrm{f})}$ using the Drude-free electron theory to show the dispersion of the surface waves $(\omega, \mathrm{k})$ and to obtain the energetic parameter $\beta$ and the wave-number parameter $\chi$ using a new identity. The graph illustrated in Fig. (6) describes the dispersion relation $\mathrm{k}$ against $\mathrm{h} \omega$. The values of the $\mathrm{K}$-vector of the surface waves are obtained from the estimated resonance angle $\alpha$ of the measured ATR-resonance signals, Fig. (1) using the formula: $[6,8,9,14]$.

$$
\mathrm{k}=\frac{\omega}{\mathrm{c}} \sqrt{\varepsilon_{2}} \sin \alpha
$$

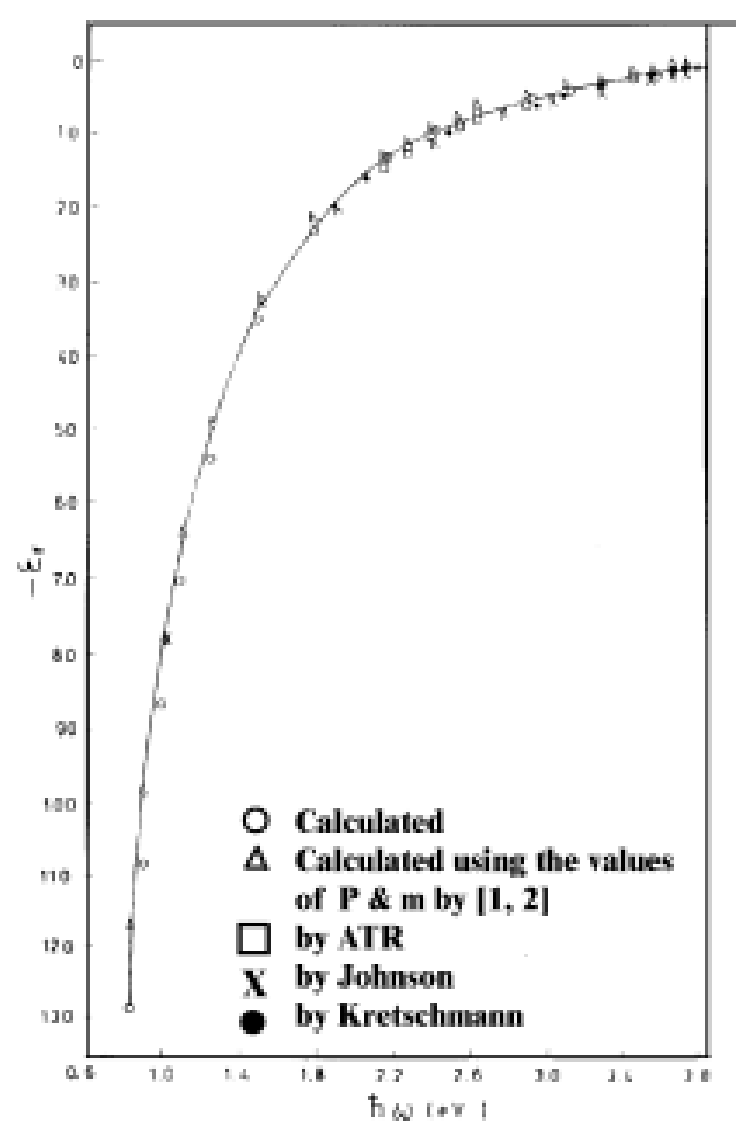

Fig. (4) The dependence of $\varepsilon_{r}$ on the photon energy.

Fig. (6) reveals the good agreement between data obtained by different authors indicating the correction of the present approach. Figures 7 and 8 show the relation between $\Delta \mathrm{k}$ the shift of the dispersion curve from the non interacting photon line $(\mathrm{k}=\omega / \mathrm{c})$ - and $\hbar \omega$ as well as between $\ln (\Delta \mathrm{k})$ against $\hbar \omega$ wherein a new identity could be valid [9]

$$
\Delta \mathrm{k}=\chi \exp (\hbar \omega / \beta) .
$$

It was found that $\beta=0.513 \mathrm{eV}, \chi=100 \mathrm{~cm}^{-1}$. The given values of $\beta, \chi$ for $\mathrm{Ag}$ films are the same either by using the free electron model or using ATRtechnique indicating the physical sense of the Drude free electron theory. The mentioned values of $\beta, \chi$ are listed in Table (1). 


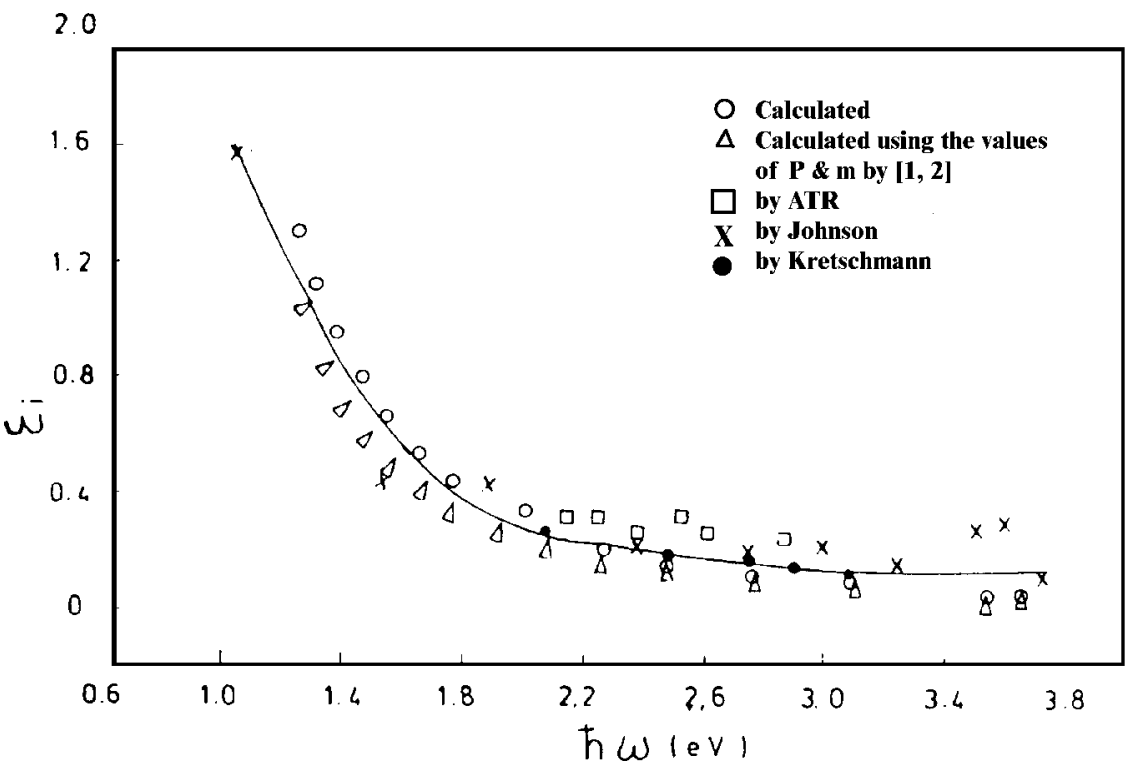

Fig. (5) The dependence of $\varepsilon_{\mathrm{i}}$ on the photon energy.

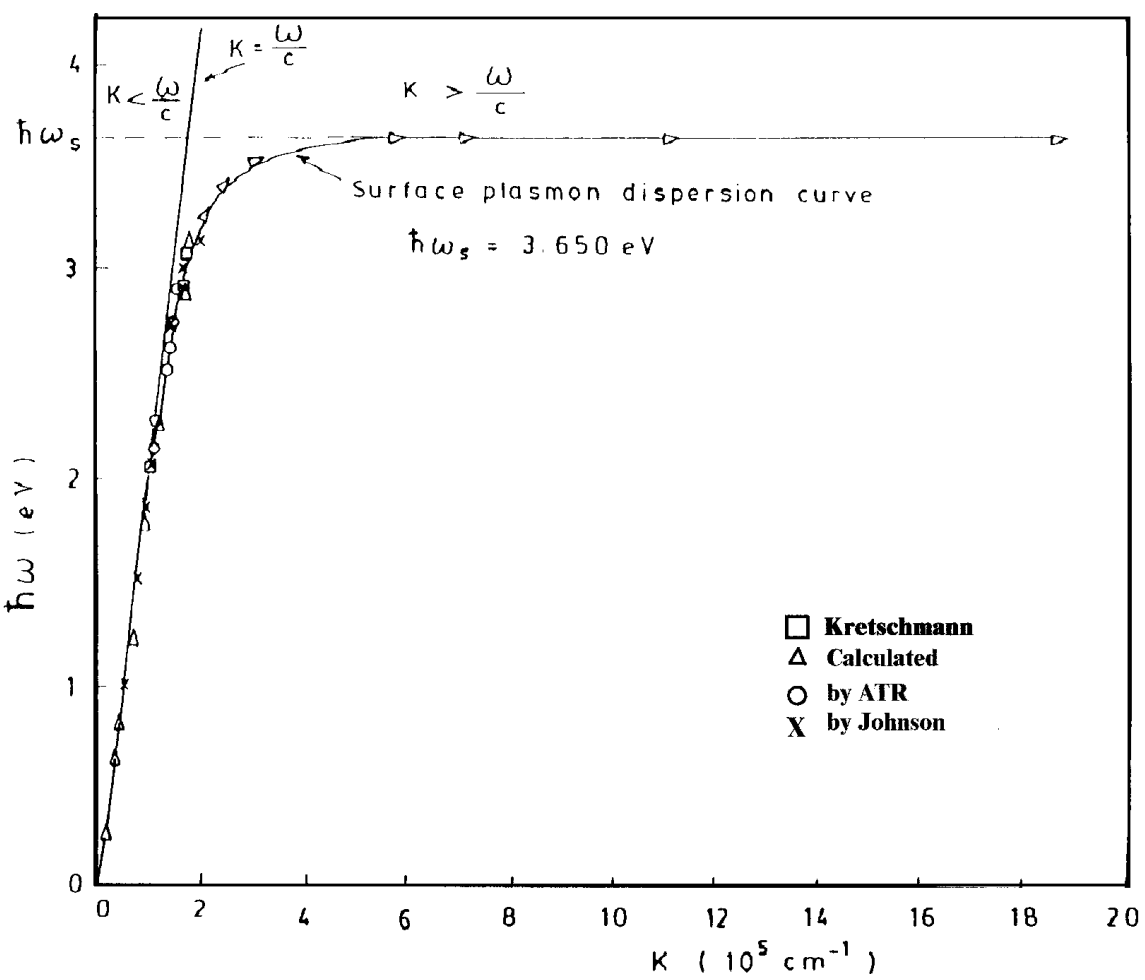

Fig. (6) The dispersion curve for the boundary Ag/air. 


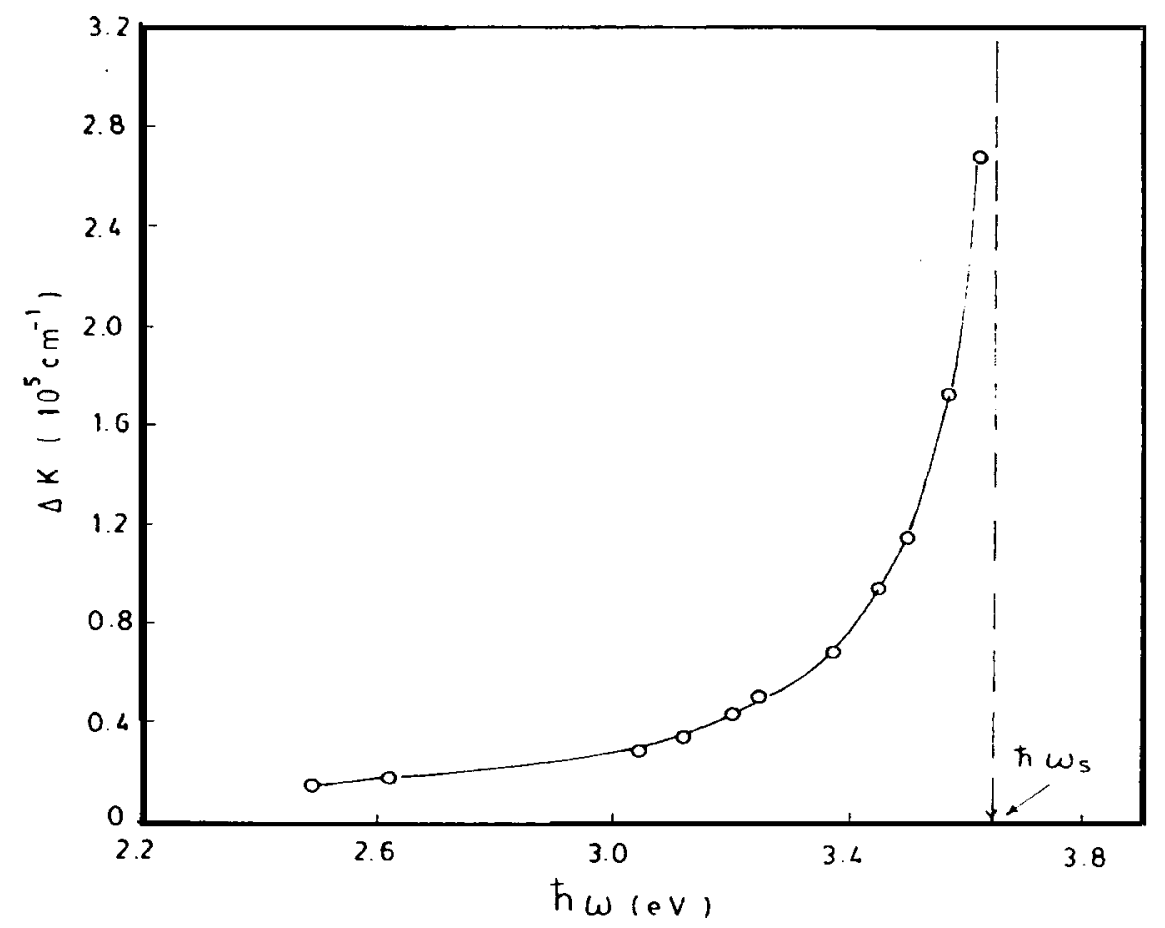

Fig. (7) $\Delta K$ against $\hbar \omega$ for the boundary $A g / a i r$.

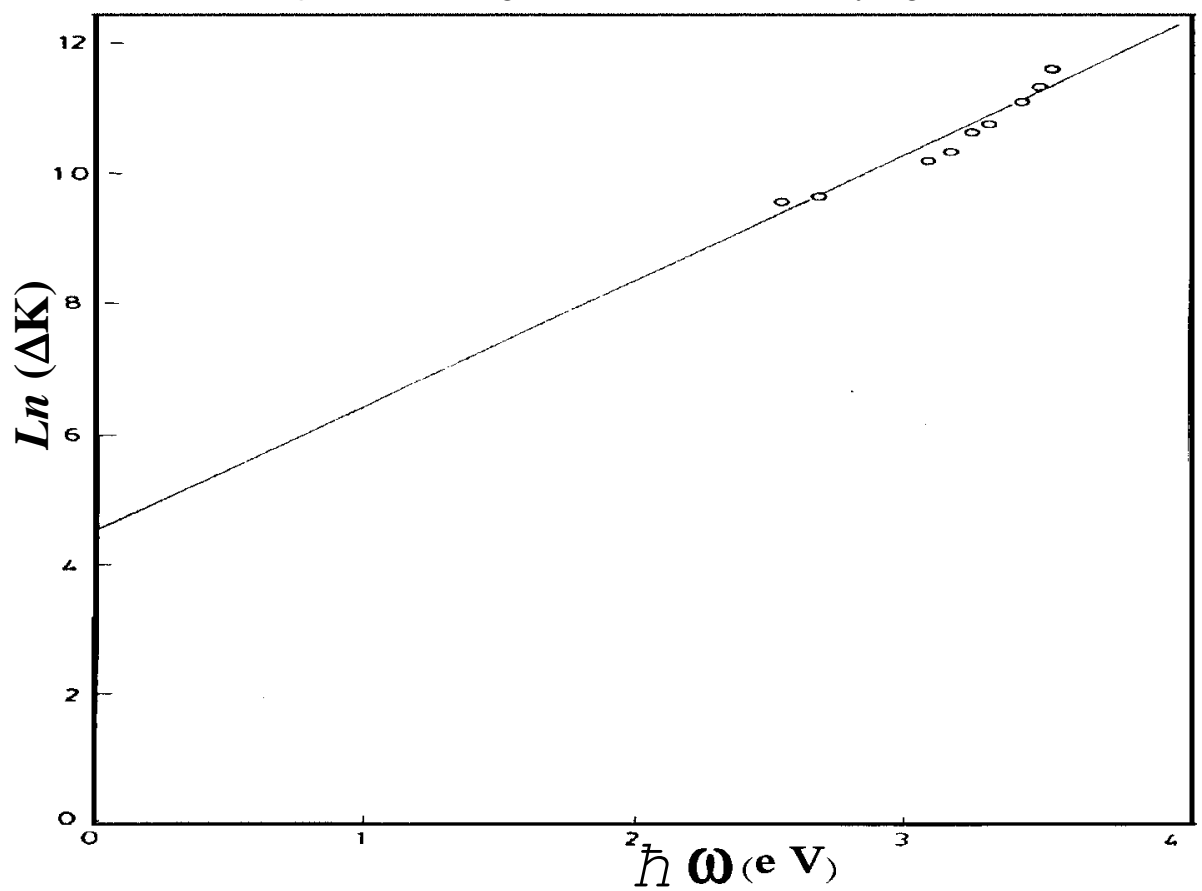

Fig. (8) $\ln (\Delta \mathrm{K})$ against $\hbar \omega$ for the boundary $\mathrm{Ag} /$ air. 
It was found that $\beta=0.513 \mathrm{eV}, \chi=100 \mathrm{~cm}^{-1}$. The given values of $\beta, \chi$ for Ag films are the same either by using the free electron model or using ATRtechnique indicating the physical sense of the Drude free electron theory. The mentioned values of $\beta, \chi$ are listed in Table (1).

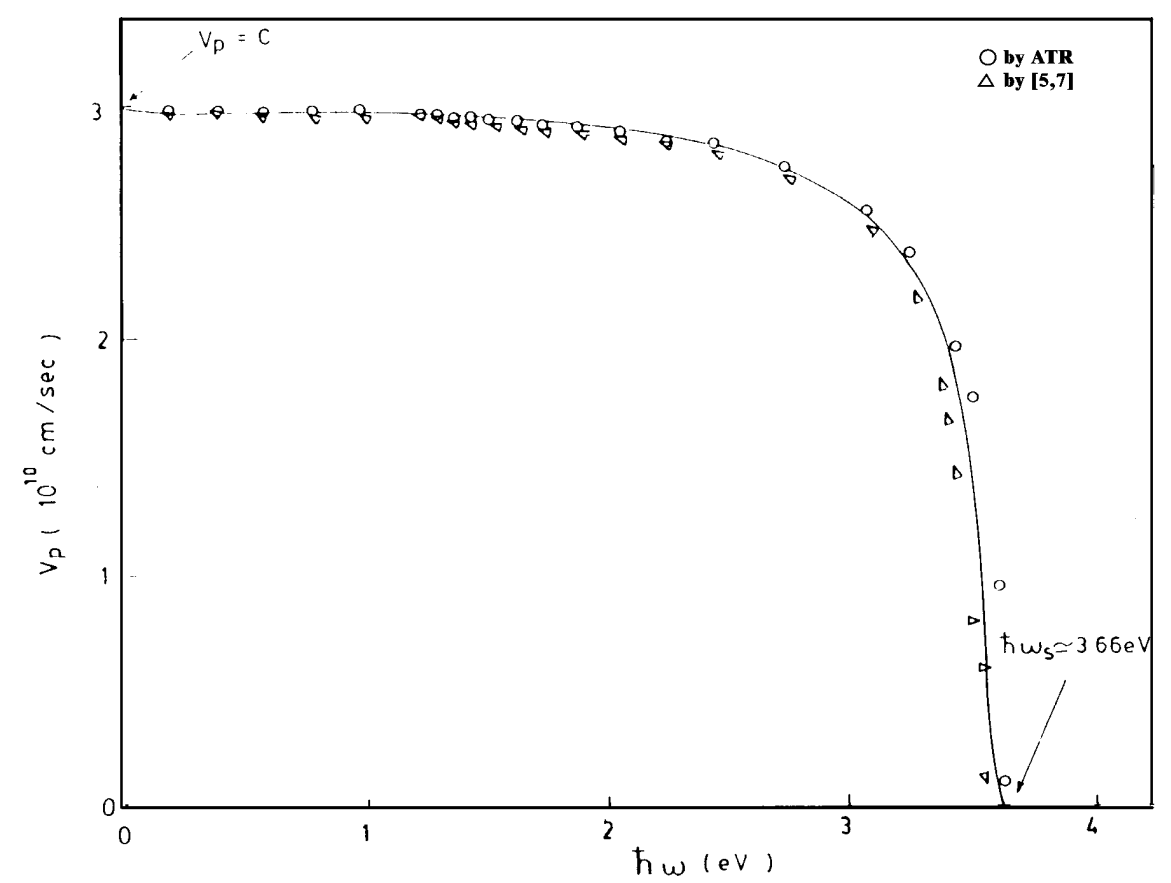

Fig. (9) $V_{p}$ against $\hbar \omega$ for the boundary Ag/air.

Since $\Delta \boldsymbol{K}$ is the shift of the non interacting photon line $(\mathrm{k}=\omega / \mathrm{c})$ from the dispersion curve - which is a measurable quantity for the interaction of photons with the surface plasma waves - as well as is usually accompanied with damping mechanism of the surface waves - [6,8], $\Delta \mathrm{k}$ is then a measurable quantity for the damping of the surface waves wherein a high values of $\Delta \mathrm{k}$ indicating enhancement of the damping process [9]. In addition, the exponential increase of $\Delta \mathrm{k}$ by increasing $\hbar \omega$ is accepted since the fields produced by the surface charges are highly concentrated at the boundary Ag/air and decay exponentially into silver and air respectively $[1,4]$. The damping mechanism is discussed in some details $[6,8]$. It could be then concluded that the exponential increase of $\Delta \mathrm{k}$ against $\hbar \omega$ Fig. (7) - leading to a growth of the damping process - corresponds to a decrease of both the phase - and group - velocity of the surface waves by increasing $\hbar \omega$ as well as the limit surface plasma energy $\hbar \omega_{\mathrm{s}}$ corresponds to a drop of both $\mathrm{V}_{\mathrm{p}} \& \mathrm{~V}_{\mathrm{g}}$ to zero Figs. ( $9 \& 10$ ), which will be discussed in some details. 


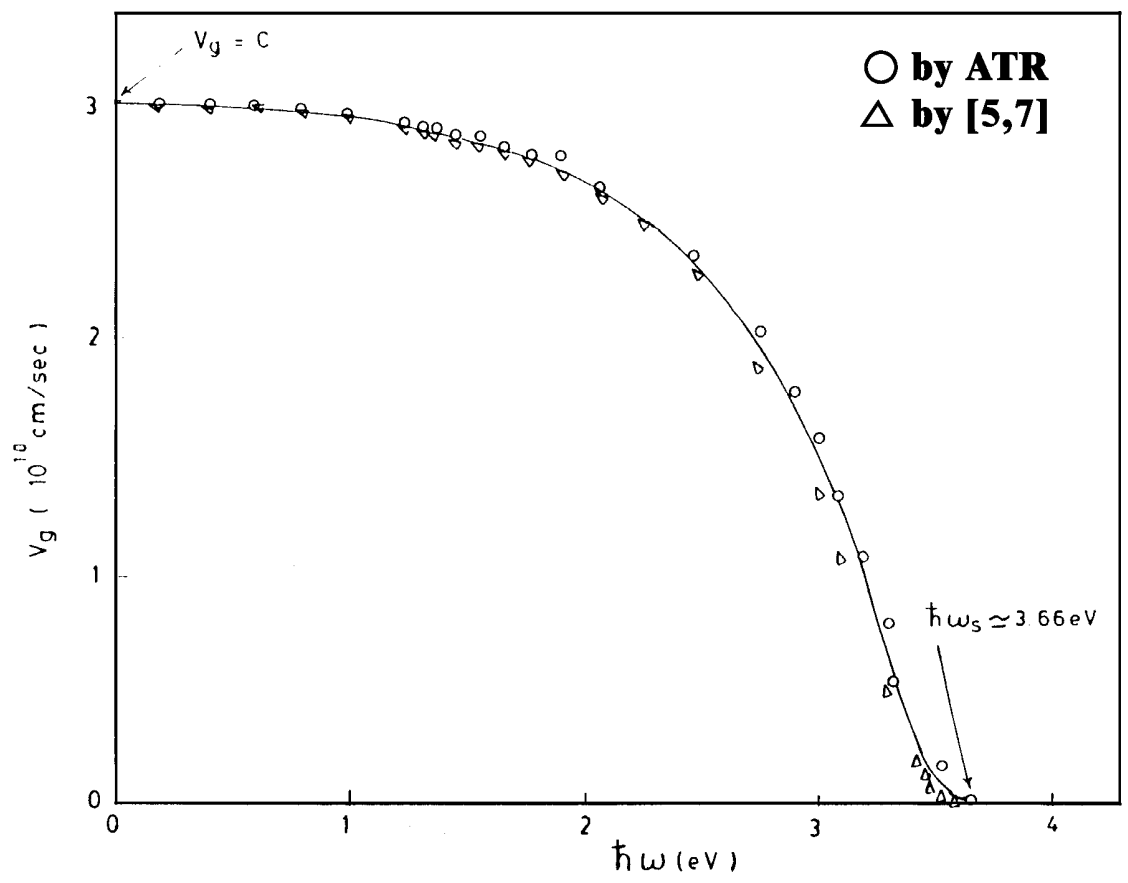

Fig. (10) $V_{g}$ against $\hbar \omega$ for the boundary Ag/air.

\section{2- Mechanism of the propagation of the surface waves:}

It is known that, the surface plasma oscillations - are collective oscillations of the charges at a metal dielectric boundary which propagate along the interface as a plane magnetic waves $[1,6]$ - as well as they are nonradiative waves transport no energy away from the boundary $[1,14]$, consequently these waves have a maximum amplitude at the surface boundary. In order to derive two formulae for the phase - and group - velocities of the surface waves, we must first of all consider the monochromatic waves which are never strictly realized in practice [15]. It is demonstrated [15] that for the superposition of plane monochromatic waves, the planes of constant amplitude and in particular, the maximum of the amplitude are propagated with the group velocity as a differential coefficient $V_{g}=d \omega / d \mathbf{k}$, whilst, the planes of constant phase are propagated with the phase velocity $\mathrm{V}_{\mathrm{p}}=\omega / \mathbf{k}$.

In a previous article a suggested formula for the dispersion relation of the surface waves $(\omega, \mathrm{k})$ for the boundary metal/air [9] is given as:

$$
\mathrm{K}^{2}=\frac{\omega^{2}}{\mathrm{c}^{2}} \cdot \frac{\left|\varepsilon_{\mathrm{r}}\right|}{\left|\varepsilon_{\mathrm{r}}\right|-1}
$$




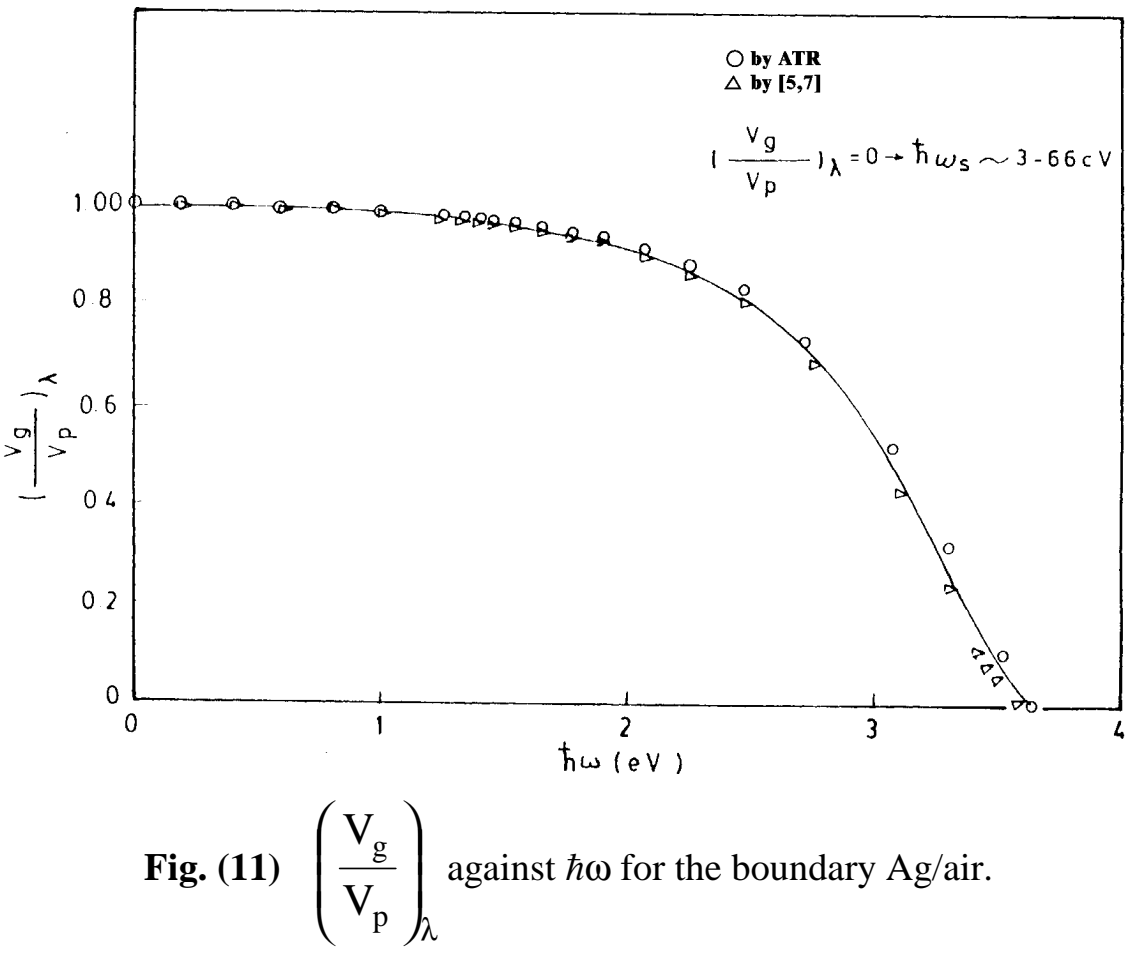

Now we derive two formulae for the phase - and group - velocity of the surface waves by applying the Drude-free electron theory taking into account the polarization effect - described by $\mathrm{P}$ - due to the interband transitions [5,7], since:

$$
\varepsilon=\mathrm{P}-\frac{\omega_{\mathrm{p}}^{2}}{\omega(\omega+\mathrm{i} / \tau)}=\varepsilon_{\mathrm{r}}+\mathrm{i} \varepsilon_{\mathrm{i}}
$$

For metals, at the visible and near - infrared frequencies, $\omega>>1 / \tau$ [7], the real and the imaginary - parts of the dielectric constant are then given as:

$$
\begin{aligned}
& \left|\varepsilon_{\mathrm{r}}^{\mathrm{f}}\right|=\frac{\omega_{\mathrm{p}}^{2}}{\omega^{2}+(1 / \tau)^{2}}-\mathrm{P}=\frac{\omega_{\mathrm{p}}^{2}}{\omega^{2}}-\mathrm{P} \\
& \varepsilon_{\mathrm{i}}=\frac{\omega_{\mathrm{p}}^{2} \tau}{\omega\left(1+\omega^{2} \tau^{2}\right)}=\frac{\omega_{\mathrm{p}}^{2}}{\omega^{3} \tau}
\end{aligned}
$$


since $\mathrm{V}_{\mathrm{p}}=\frac{\omega}{\mathrm{K}}, \varepsilon_{\mathrm{r}}=\varepsilon_{\mathrm{r}}^{\mathrm{f}}$, one obtains

$$
\mathrm{V}_{\mathrm{p}}^{2}=\frac{\mathrm{c}^{2}\left(\omega_{\mathrm{p}}^{2}-\omega^{2} \mathrm{P}-\omega^{2}\right)}{\omega_{\mathrm{p}}^{2}-\omega^{2} \mathrm{P}}
$$

In addition, Since

$$
\mathrm{K}^{2} \mathrm{c}^{2} \omega^{2}=\left(\mathrm{K}^{2} \mathrm{c}^{2}-\omega^{2}\right)\left(\omega_{\mathrm{p}}^{2}-\omega^{2} \mathrm{P}\right)
$$

Hence by the differentiation of $\omega$ with respect to $K$ wherein $V_{g}=d \omega / d k$, it follows:

$$
V_{g}^{2}=\frac{V_{p}^{6}\left(\omega^{2} P-\omega_{p}^{2}\right)^{4}}{C^{4}\left(\omega^{4} P-2 \omega^{2} \omega_{p}^{2} P+\omega^{4} P^{2}+\omega_{p}^{4}\right)^{2}}
$$

As has been demonstrated in the previous section, the volume plasma frequency $\omega_{\mathrm{p}}$ as well as the polarization parameter $\mathrm{P}$ are obtained and listed in Table (1). In addition from eq. 3 for the boundary metal/air the surface plasma frequency $\left(\left|\varepsilon_{\mathrm{r}}\right|=1\right)$ is given by: $\omega_{\mathrm{s}}=\omega_{\mathrm{p}} / \sqrt{1+\mathrm{P}}$, which is the limit frequency for the excitation of the surface waves. It has been demonstrated [16] that in the region between $\omega_{\mathrm{s}}$ and $\omega_{\mathrm{p}} / \sqrt{\varepsilon_{\mathrm{O}}}$ - where $\varepsilon_{\mathrm{O}}$ is the adjacent medium, in our case is the air $\varepsilon_{\mathrm{o}}=1$ - no surface modes can be excited. We have then restricted our calculations in the spectral range: $\omega \leq \omega_{\mathrm{s}}$. Consequently, for the boundary Ag/air $\hbar \omega_{\mathrm{p}}=9.57 \mathrm{eV}$ and $h \omega_{\mathrm{s}}=3.66 \mathrm{eV}$. Also from eq. 3 at $\varepsilon_{\mathrm{r}}^{\mathrm{f}}=0, \omega_{\mathrm{i}}=\omega_{\mathrm{p}} / \sqrt{\mathrm{P}}$, $\hbar \omega_{\mathrm{i}}=3.974 \mathrm{eV}$ which corresponds to the onset of interband transitions which is in the range $3.77-4 \mathrm{eV}[5,7,17,18]$, in accordance with our extracted value indicating that the value $\mathrm{P} \sim 5.8$ is acceptable.

The extracted values of the optical quantities $\mathrm{P}, \omega_{\mathrm{p}}^{2}, \mathrm{~m}_{\mathrm{o}}, \tau, \beta$ and $\chi$ for silver are listed in Table 1.

Table (1)

Ag-films d $\sim 50 \mathrm{~nm}, \mathrm{~h} \omega_{\mathrm{S}}=3.66 \mathrm{eV}, \mathrm{h} \omega_{\mathrm{i}}=3.974 \mathrm{eV}$.

\begin{tabular}{|c|c|c|c|c|c|}
\hline $\mathrm{P}$ & $\omega_{\mathrm{p}}^{2} \times 10^{32} \mathrm{~s}^{-2}$ & $\mathrm{~m}_{\mathrm{o}}$ & $\tau \times 10^{-14} \mathrm{sec}$ & $\beta(\mathrm{eV})$ & $\left.\begin{array}{c}\chi\left(\mathrm{cm}^{-}\right. \\
1\end{array}\right)$ \\
\hline 5.8 & 2.123 & $0.90 \mathrm{~m}$ & 2.424 & 0.513 & 100 \\
\hline
\end{tabular}


Now, we have calculated $V_{p}, V_{g}$ and $\left(V_{g} / V_{p}\right) \lambda$ using eqs. $(6 \&$ \% $)$ utilizing our mentioned calculated values $\omega_{\mathrm{p}}^{2}, \tau, \mathrm{P}$ as well as the values obtained by [5,7]. The vlaues of $\mathrm{V}_{\mathrm{p}}, \mathrm{V}_{\mathrm{g}}$ and $\left(\mathrm{V}_{\mathrm{g}} / \mathrm{V}_{\mathrm{p}}\right) \lambda$ are shown in Figs. (9-11) against the photon energy $h \omega$. It is observed that our values are in consistent with that obtained by $[5,7]$. Furthermore our calculated value $\mathrm{P} \sim 5.8$ leads to the limit surface energy $\hbar \omega_{\mathrm{s}}=\frac{\hbar \omega_{\mathrm{p}}}{\sqrt{1+\mathrm{p}}} \sim 3.66 \mathrm{eV}$ which corresponds to $\mathrm{V}_{\mathrm{p}}=\mathrm{V}_{\mathrm{g}}=0$ as illustrated in Figs. ( $9 \& 10)$ which is in accordance also with eqs. $(6 \& 7)$. This phenomena corresponds to infinite values of the K-vector of the surface waves as shown in Fig. (6), as well as infinitesimal values of both $\mathrm{V}_{\mathrm{p}}, \mathrm{V}_{\mathrm{g}}$ of the surface waves-around the spectral region $\omega \sim \omega_{\mathrm{s}}$ - which are nonradiative waves transport no energy away from the surface boundary [1]. Moreover these waves are then localized waves as indicated by $[6,19]$.

From the above demonstrations, the free electron model is very useful describing the characteristic behavior of the localized transverse magnetic surface waves [6] at the boundary Ag/air-taking into account the polarization effect due to the interband transition, as well as, for estimating the optical constants with good accuracy.

It should be noticed that for low photon energies an increase of both $V_{p}$ and $\mathrm{V}_{\mathrm{g}}$ is observed Figs. $(9,10)$ as well as they tend to the velocity of light at zero frequency in which the medium is nondispersive $[15,20]$ which is in consistent with the analysis obtained by [15] in which for a nondispersive medium the phase- and the group - velocity are both equal.

\section{Conclusion}

Using the free electron theory, a general identities could be used to estimate the real - and the imaginary - part of the dielectric constant $\varepsilon_{\mathrm{r}}^{\mathrm{f}}, \varepsilon_{\mathrm{i}}^{\mathrm{f}}$ for silver films with good accuracy using the estimated values of the polarization parameter $P$ as well as the volume plasma frequency $\omega_{p}$, the relaxation time $\tau$ of the electrons. Also the values of the energetic parameter $\beta$ and the wavenumber parameter $\chi$ could be obtained with good accuracy. Moreover, a derivation of two formulae of the phase- and the group - velocity of the surface waves could be obtained. 


\section{Acknowledgement}

The authors are grateful to Prof. Dr. H. Talaat, Ain Shams University for fruitful discussions and critical reading of the manuscript as well as Prof. Dr. M. M. El-Ocker, for his kind advice and encouragement, where our experimental work has been carried out at "The Laboratory for Optical Measurements of Solids," Physics Department, Faculty of Science, Al-Azhar University, Cairo.

\section{References}

1. H. Raether, in: Physics of thin films, 9, 145 (1977).

2. E.A. Stern and R.A. Ferrell, Phys. Rev. Vol. 120, [1], 130(1960).

3. B.O. Seraphin (Optical Properties of Solids), New Developments, American Elsevier Publishing Company, Inc., New York, page 678 (1976).

4. A. Otto, Sektion Physik der Universitat Munchen, Germany, Festkorperprobleme XIV, page 1-36 (1974).

5. F. Abele's, (Optical Properties of Solids), North-Holland Publishing Company - Amsterdam, London, 74-132 (1972).

6. A. Otto, Z. Physik, 216, 398 (1968).

7. P.B. Johnson and R.W. Christy, Phys. Rev B Vol. 6, no. 12, 4370 (1972).

8. E. Kretschmann, Z. Physik 241, 313 (1971).

9. M.M. El-Ocker and A.M. Gadou, Solid state communications, 76, [11], 1297 (1990).

10. A.M. Gadou, Ph.D.Thesis, Faculty of Science, Al-Azhar University, 1988.

11. N.W. Ashcroft, N.D. Mermin, (Solid State Physics) Library of Congress Cataloging in Publication Data, New York, 5, 827 (1976).

12. K.F. Riley: Mathematical Methods for the Physical Sciences, Academic Press Inc., New York, page 22, 1974.

13. Ehrenreich and H.R. Philipp, Phys. Rev. 128, [4], 1622 (1962).

14. I. Pockrand, J.D. Swalen, R. Santo, A. Brillante and M.R. Philpott, J. Chem. Phys. 69, 4001 (1978).

15. M. Born and E. Wolf, Principles of Optics, Pergamon Press, Oxford, London, New York, Chapter 1 (1975).

16. S.O. Sari, D.K. Cohen and K.D. Scherkoske, Phys. Rev. B, 21, [6], 2162 (1979).

17. E.A. Taft and H.R. Philipp, Phys. Rev. 121, [4], 1100 (1961).

18. P.O. Nilsson, I. Lindau, and S.B.M. Hagstrom, Phys. Rev. B, 1, [2], 298 (1970).

19. M.J. Jory, G.W. Bradberry, P.S. Cann and J.R. Sambles Measurements Science and Technology, 6, [8], 1193, August (1995).

20. R.D. Guenther, Modern Optics, John Wiley \& Sons, New York, (1990). 